\title{
Learning Management System based on Machine Learning: The Case Study of Ha'il University - KSA
}

\author{
Mohamed Hédi Maâloul $^{1}$, Younès Bahou ${ }^{2}$ \\ Computer Science Department, Community College - University of Ha'il \\ Ha'il - Kingdom of Saudi Arabia
}

\begin{abstract}
Online learning environments have become an established presence in higher education in the Kingdom of Saudi Arabia, especially with the expected of Covid-19 pandemic. At present, supporting e-learning with interactive virtual campuses is a future aim in education. In order to solve the problems of the interactivity and the adaptability of e-online learning systems in Saudi universities, this paper proposes a module, based on digital learning, and to be used in learning management systems to meet the challenges a future goal in e-online learning. The e-learning system should be intelligent and has the possibility to inspire the specific characteristics (i.e., metadata) of a student used to access to their social media profiles.
\end{abstract}

Keywords-Learning management systems; blackboard; machine learning; semi-supervised learning; personalization system; SPS System; user profile; social profile

\section{INTRODUCTION}

In this exceptional pandemic situation related to Covid-19, educational activity was affected to different degrees during containment (closures, activity limitations, reorganizations, etc.). The progressive resumption of activity towards a stabilized situation raises many questions and requires a preparation facilitating its conditions of success.

Therefore, total e-learning has become an obligation for higher education worldwide and particularly in the Kingdom of Saudi Arabia. The Blackboard is the system used at the University of Ha'il to achieve the e-learning aims.

Many types of learning platforms (e.g., Blackboard, Formare, Moodle, Teleformar, WebCT, etc.) have been considered as an opportunity for Saudi universities to provide online courses, tests and evaluations, databases and for online monitor students' progress [1,2].

However, these courses are stored as files on the Learning Management Systems (LMS) server as a single course. These courses do not take into consideration the user's profile (i.e., student's profile) and prerequisites.

This work falls within the framework of improving the performance of learning platforms and the Blackboard platform, and more precisely at the level of the student profile definition module.

Thus, our goal is to experiment with a numerical approach, not currently implemented in the Blackboard platform.

This paper is structured around four sections. This paper is structured around four panels.
The first section presents literature on LMS and the effectiveness of these learning systems, particularly with the expected Covid-19 pandemic.

The second part presents the proposed method for defining a student profile based on the information contained in his social network. The student profile is useful for any learning system that aims to adapt and propose appropriate content that corresponds to a student's specific needs.

The third part presents the Social Profiling of the Student (SPS) system and the process of generating a student's (i.e., social profile).

Finally, the last part focuses on the implementation and evaluation of the proposed method.

\section{LITERATURE}

Smartphones, tablets, and computers are essential tools in the educational process especially after the spread of Covid-19 all around the world. In the Middle East, and more specifically the University of Ha'il in the Kingdom of Saudi Arabia, the use of IT tools is increasing constantly among students at universities.

Hence, platforms, websites and e-learning Systems are essential and the best way to save the learning process during Covid-19. According to A. N. Alkhaldi, and A. M. Abualkishik, the previous studies mentioned that the Blackboard is a recent technological tool that contributes to sharing knowledge, making quizzes, taking attendance and evaluating students [6].

The earlier study showed that Blackboard "was founded in 1997 by two education advisors, Matthew Pittinsky and Michael Chasen, as a consulting firm to provide technical standards for online learning applications" [17]. They added that the Blackboard witnessed a huge rise until it reached $\$ 752$ million in basic stock and become public in June 2004.

In [4] before 2500 years, memorization was the essential method of teaching and the favourable way to preserve knowledge and skills, but the communication was usually oral.

After that, technology has been used in teaching and learning for many years. The results of using technology in teaching represent that there is a positive influence on the education process.

Universities and educational institutions try to improve their own LMSs in order to provide their smart learning online aspects for different groups of learners. The recent studies 
define LMSs as the web-based system that provides many benefits for the educational processes [14].

LMS can be used as an effective tool for students belonging to the same University and studying in different campuses [9]. LMS is used to assemble students from a different place and different time zone to join the same lecture, to solve the same problem, to do the same quiz or to sit for the same exam.

According to L. A. Bove and S. Conklin (2020), LMS can simplify and speed the discussions, document sharing, and assignment submission between teachers and their students [13]. Moreover, it helps teachers to better evaluate their students.

Consequently, LMS is helpful and useful for students who witnessed difficulties to move from their home town or home countries to join the lectures at universities. Additionally, LMS is gainful for special needs students.

The cultural aspects of LMS design are of huge importance because it can fit the user's satisfaction which can provide success stories in education for both students and teachers. In addition, learning materials on LMS can respect gender differences. Next, LMS can afford guidelines for students and build a learner-centred educational climate [9].

Today, mobile applications make the use of LMS easier and painless. Students can access their LMS accounts despite the places they are in such as trains, buses, homes, etc.

Mobile Apps are considered as a recent infrastructure that contributes to the spread of LMS. A. W. Bates (2015) in [8] argued that the social media is a significant technology which supports the teaching method since it facilitates the dialogues and strengths the students' contribution during the lectures.

The Blackboard advances since its foundation until now. The benefits of the Blackboard Learning System are the increased availability, the quick feedback, the improved communication, the tracking, and the skills building [12].

The Digital Report (2020) in Saudi Arabia (https : / / datareportal.com/reports/digital-2020-saudi-arabia) mentioned that the number of social media users rise by $(8.7 \%)$ between April 2019 and January 2020 (about 2 million users). The report shows that the most used social media platforms are YouTube (76\%), WhatsApp (71\%), Instagram (65\%), Facebook (62\%), and Twitter (58\%).

However, the statistics show that the preferable social network for students in the University of Ha'il is WhatsApp [7]. Therefore, we build our study on the consideration of the WhatsApp as the first source of data for the User Profile.

Despite the huge number of the users of social media platforms, students cannot access to the Blackboard through their social networking information. Nevertheless, the use of the data from the social network let students butter manage their Blackboard accounts.

The previous studies tried to count different drawbacks of the Blackboard. They stated that the Blackboard is hard to learn, its options may be restricted to particular operating systems, its system inefficiencies, and the cost of the Blackboard is really high [17].

In the past, one of the key limitations of an LMS is the dilemma of how to use new technology in the learning process [5].

There are two challenges in online learning the first is to provide the students with new technological knowledge, the second is to attract their attention to follow the main lecture. The serious obstacle that faces the teacher is that students are not motivated to become expert users of LMS [16].

As a result, in the current study, we try to make the LMS easier for the students at the University of Ha'il through the integration of students' data from social networks.

In defiance of the advances it witnessed, the LMS Blackboard still faces a serious drawback that the previous studies did not deal with it: the user profile, this aspect is the one we will work in in the study in hands.

\section{A. e-Online Learning Challenges}

The LMS Blackboard is a standard system; it witnessed some development, but they are not in-depth. Despite the validation of advancement on the Blackboard, the LMS is still incapable to manage the student's metadata from other platforms; it cannot capture student parameters from WhatsApp, Twitter, Facebook, etc.

The present study works on the development of the integration of the student's email used in social networks to access to the Blackboard, instead of using their University Id number (e.g., s20200123). Hence, the student can relate his Blackboard account with their social media profiles.

It is hard for a student to use his preferences; they can forget their Id Number or type it in a wrong way because they use it only to access to the Blackboard or during the exam days. Hence, it is difficult for students to use preferences to access to the Blackboard LMS system, so we are working in the current study to facilitate their access into the LMS by using things with is easy to remember their social media parameters since they use it daily and this step aims to improve the effectiveness of the educational process.

The LMS Blackboard cannot configure student's settings which are fixed by the University technical Unit. Even when the student's name is written in the wrong way, he/she cannot correct it or make any changes. As a result, the University technical unit is supreme power when dealing with the Blackboard, whereas the educational process should be based on the student.

\section{B. Concept of user Profile}

The notion of the user profile is widely addressed in user modelling, which can be considered as the process of knowledge extraction in order to identify the information and characteristics of the user or a group of users [3].

The use of the user model in this work improves the quality of human-machine interactions: the deduction of user preferences and contexts from the activities observed in the 
social media is used to determine the type of dialogue that the LMS system will have with the user.

Indeed, the user profile is used to adapt and propose appropriate content that corresponds to the user's specific needs [18].

Our work deals with the definition of the student profile which aims to allow the LMS system to adapt to the user. The following section explains this aspect, in the context of information customization systems.

Our study is used to determine, in a first place, the personal data of students that are relatively stable over time, such as their identity (e.g., e-mail, phone number), demographic data (e.g., age, gender, address) and that do not require automatic updates.

Secondly, we consider that preferences and interests tend to change over time and that designate the characteristics of the user such as preferred types of presentation, etc.

In this context, our contribution is to propose a recommendation system that is fundamentally based on a digital learning-based technique (i.e., semi-supervised learning) and that determines the degree of similarity between students, in order to recommend the items corresponding to a student's interest.

The choice of the machine learning technique based on semi-supervised learning is justified by the fact that it allows involving a system with only a small number of labelled items students and a large number of not labelled ones.

The method that we present in this paper aims to process student profiles from the e-learning platform. To do so, we have proposed a digital learning approach [20].

\section{PRoposed Methodology}

Our proposal aims to predict and determine the preferences of the student from the information shared in his different social media. This extracted knowledge allows us to build the student profile that becomes central in any LMS system.

We propose a new method for using the user profile (i.e., student profile) in a referral system to provide a student with resources (i.e., content and items) relevant to his or her interests or specific needs in social media.

Our method is based on a machine learning technique. More precisely, it is based on the semi-supervised learning technique which is composed of two phases: the first one is the learning phase which allows the system to learn how to provide a student with personalized resources [15,21]. We use the J48 algorithm for this phase. The choice of using the J48 algorithm is justified by the fact that it has given the best Fmeasure scores in comparison with the classification algorithms.

The second phase is the use phase, which allows users to use the learning platforms. Fig. 1 shows the details of the proposed method and the two phases (i.e., the learning phase and the use phase).

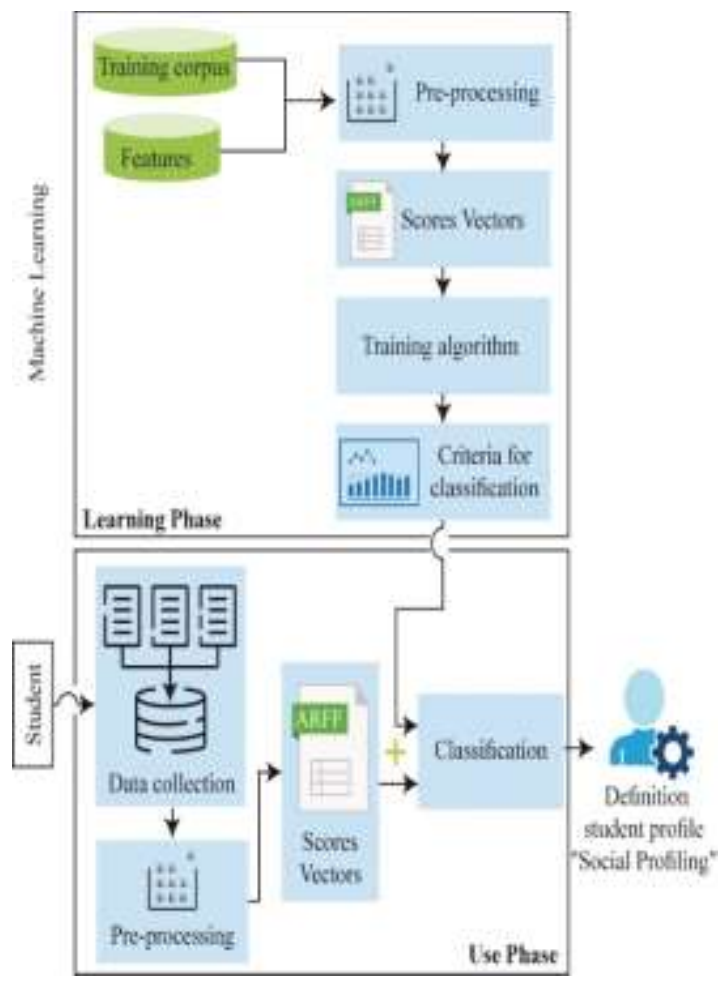

Fig. 1. Principle of the Proposed Method.

\section{A. The Learning Phase}

In this phase, we provide a training corpus and metadata features reflecting the student's interests. This information is defined following several studies [7].

The training corpus is composed of the attributes assigned to student information and their profiles. Attributes are assigned to student information and come from different categories: demographic data (e.g., age, gender, address), interests, hobbies, affiliation, preferences [19].

All attributes are initially pre-processed to prepare their segmentations in gender (i.e., male or female), age, description or structured as vectors (e.g., interests). However, some attributes have only one value (e.g., age, gender) while others may have several possible values (e.g., favourite music groups, favourite sports, etc.).

After the pre-processing step, each student entity (i.e., attribute) will be notified according to certain metadata characteristics. This step leads to the construction of a set of $\mathrm{V}$ vectors corresponding to the values of the specific characteristics (i.e., metadata) of a student. These vectors are called score vectors. Each vector is associated with classes and indicates the class of the student node.

The score vector has the following structure, V1 (A1, A2, A3... An), where Ai is the score of the metadata criteria from $\mathrm{i}$ to $n$ (i.e., the number of criteria).

In the learning phase, the score vectors are combined to associate a score to each characteristic and to generate classification rules. 


\section{B. The use Phase}

This step is based on the construction of a student profile from the information contained in his or her social network, which we call "social profiling".

The term "social profile" refers to a profile constructed using the student's social network.

A social profile contains metadata reflecting the student's interests extracted from the information shared by the individuals in his or her social network.

Our objective in this phase is to build from this shared information on the student's social network, a score vector based on affinities (e.g., geographical, affiliation, interests, etc.).

Thus, we start this second phase with a step of collecting the student's shared information in his social network. An important point here is data collection problems, which is widely studied in social network analysis. In order to solve this problem, several APIs (Application Programming Interface) tools [11] that allow to query and process very large volumes of data related to public social networks (e.g., Twitter, Facebook, Instagram) have been applied (this is the step of collecting a student attribute).

After the pre-processing step of the collected data, and with the score vectors and the result defined by the learning phase using the $\mathrm{J} 48$ learning algorithm, which has proven its efficiency in our proposal, the "social profiling" of the student will be defined in this way.

To justify the choice of our learning algorithm, we tested five best classification algorithms according to the F-measure evaluation metric [10]. We deduced that the J48 algorithm gives the best F-measure. Table I summarizes the test results of the five algorithms.

TABLE I. ClassificATION ALgORITHM TEST RESUlts

\begin{tabular}{|l|l|l|l|}
\hline Algorithm & Precision & Recall & F-Measure \\
\hline J48 & 0.898 & 0.855 & 0.876 \\
\hline PART & 0.907 & 0.830 & 0.867 \\
\hline SVM & 0.615 & 0.609 & 0.588 \\
\hline BayesNet & 0.570 & 0.566 & 0.565 \\
\hline NaiveBayes & 0.542 & 0.545 & 0.538 \\
\hline
\end{tabular}

\section{SPS SYSTEM}

The method we proposed for the automatic definition of social profiling of the student was implemented through the SPS system. In this section, we present the implementation details and preliminary results.

\section{A. Implementation Details}

Our study corpus consists of 427 student nodes collected from a survey questionnaire that was used to collect data from students at Ha'il University in Saudi Arabia and is accessible to the Blackboard system [7].
Using the study conducted by A. N. Alkhaldi, M. Ali, S. M. Mahmoud, Z. A. Alrefai, and Y. Bahou (2020), we were able to draw up a list of "features" criteria defining the links between students according to their common points or affinities (i.e., geographical, affiliation, interests) [7].

Note that the implementation requires a pre-processing step. This is the preparation of the data. Preparation is the process of collecting, combining, structuring, and organizing data so that it can be analysed in data visualization, analysis, and machine learning applications.

After the data preparation "pre-processing" step, we use nine "features" criteria to classify and define the metadata of each student entity.

Finally, we obtain a file that contains all the score vectors that make up the contribution of each student entity to learning. Table II shows features details.

In the learning phase, we use the J48 algorithm to learn how to classify the student entities. At the end of the learning phase, a score is associated with each element. Some features can have a grade of zero. The J48 algorithm generates a rule by summing the scores associated with each student entity. The system uses the generated rules to calculate the score for each student entity.

Finally, the system combines the classification decisions to obtain a social profile of the student. A social profile contains the metadata reflecting the student's interests extracted from the information shared in their social network and classified by the result generated by the $\mathrm{J} 48$ algorithm.

\section{B. Preliminary Results}

We used 427 student entities from our corpus to experiment our system (377 student nodes for the learning phase and 50 student nodes for the evaluation phase). The summaries obtained are compared to the expert results.

The main measures for Precision, Recall and F-Measure are $0.943,0.961$ and 0.952 , respectively (see Table III).

\section{TABLE II. FEATURES DETAILS}

\begin{tabular}{|c|c|}
\hline Features & Details \\
\hline Stu_Sex & Indicates the type of sex $(1=$ male; $0=$ female $)$ \\
\hline Stu_Diploma & Indicates the type of diploma (Diploma; Bachelor; Master) \\
\hline Stu_Speciality & $\begin{array}{l}\text { Indicates the speciality of the student (Medical and health } \\
\text { science; Humanist and social science; Applied science; } \\
\text { Natural science) }\end{array}$ \\
\hline Stu_Branch & Indicates the geographic location of the student \\
\hline Stu_Device & $\begin{array}{l}\text { Indicates using Blackboard system via (Smartphone; PC; } \\
\text { Together) }\end{array}$ \\
\hline Stu_Soc_Med & $\begin{array}{l}\text { Indicates the social media most used by students (Twitter; } \\
\text { Facebook; Instagram; Other) }\end{array}$ \\
\hline Stu_Pre_Film & Calculates the $\mathrm{tf}^{*}$ idf of the media preference (tf*idf Film) \\
\hline Stu_Pre_Song & Calculates the $\mathrm{tf}^{*} \mathrm{idf}$ of the media preference $(\mathrm{tf} * \mathrm{idf}$ Song) \\
\hline Stu_Pre_Doc & $\begin{array}{l}\text { Calculates the } \mathrm{tf}^{*} * \mathrm{idf} \text { of the media preference }(\mathrm{tf} * \mathrm{idf} \\
\text { Document) }\end{array}$ \\
\hline Stu_Language & Indicates the interface language \\
\hline
\end{tabular}


TABLE III. EVALUATION RESULTS

\begin{tabular}{|l|l|l|l|}
\cline { 2 - 4 } \multicolumn{1}{c|}{} & Precision & Recall & F-Measure \\
\hline Weighted Avg. & 0.943 & 0.961 & 0.952 \\
\hline
\end{tabular}

\section{CONCLUSION AND Future WORK}

In this document, we have proposed a method for defining a social profile that reflects the student's interests. The metadata of the social profile will be useful later on to improve the efficiency of the Blackboard learning process.

Our method is implemented by the SPS system and is based on the technique of machine learning. Indeed, our work focuses on a particular type of student nodes (i.e., Ha'il University students). We believe that the preliminary results are very encouraging. Indeed, the F-measurement is equal to 0.952 .

Note, we have used a small corpus for the evaluation and as a perspective, we plan to extend our evaluation to a larger corpus and also to study the effect of other criteria on the definition of student social profiling.

We also plan to enrich our proposal so that it is able to follow the evaluation of the student's social profile with a variety of interests and needs over time. We, therefore, propose to keep the profile up to date through profile updating techniques, which, starting from an already relevant and up-todate profile, will adjust at each update the interests considered relevant based on the former interests of the previous period [16]. It is thus a question of integrating a temporal factor (a temporal measure in the step of extraction and weighting of student interests).

We could also consider evaluating the social profiles constructed within the framework of our proposal in several LMS, a recommendation system for example the Blackboard.

\section{ACKNOWLEDGMENT}

This Research has been funded by Scientific Research Deanship at University of Ha'il - Saudi Arabia through project number RG-191341.

\section{REFERENCES}

[1] A. Bartuskova, O. Krejcar, and I. Soukal, "Framework of design requirements for e-learning applied on Blackboard learning system," Computational Collective Intelligence, Springer International Publishing Switzerland, pp. 471-480, 2015. https://doi.org/10.1007/978-3-31924306-1_46.

[2] A. H. Duin, and J. Tham, The current state of analytics: Implications for Learning Management System (LMS) use in writing pedagogy," Computers and Composition, vol. 55, article number 102544, March 2020.

[3] A. Kobsa, Generic user modeling systems, BRUSILOVSKY P., KOBSA A., NEJDL W. (dirs.), The Adaptive Web, Springer Berlin Heidelberg (Lecture Notes in Computer Science), pp. 136-154, 2007.

[4] A. Kukulska-Hulme, E. Beirne, G. Conole, E. Costello, T. Coughlan, R. Ferguson, E. FitzGerald, M. Gaved, C. Herodotou, W. Holmes, C. Mac Lochlainn, M. Nic Giolla Mhichíl, B. Rienties, J. Sargent, E. Scanlon, M. Sharples, and D. Whitelock, Innovating pedagogy 2020: Open
University innovation report 8, The Open University, Milton Keynes, 2020.

[5] A. M. Heirdsfield, S. Walker, M. Tambyah, and D. A. Beutel, "Blackboard as an online learning environment: what do teacher education students and staff think?," Australian Journal of Teacher Education, vol. 36, issue 7, 2011.

[6] A. N. Alkhaldi, and A. M. Abualkishik, "The mobile Blackboard system in higher education: Discovering benefits and challenges facing students," International Journal of Advanced and Applied Sciences, vol. 6, issue 6, pp. 6-14, 2019.

[7] A. N. Alkhaldi, M. Ali, S. M. Mahmoud, Z. A. Alrefai, and Y. Bahou, "Challenges facing students to adopting the Blackboard system: the case study of University of Ha'il in Saudi Arabia," International Journal of Advanced and Applied Sciences - IJAAS, vol. 8, issue 3, 2020.

[8] A. W. Bates, Teaching in a digital age: Guidelines for designing teaching and learning, Vancouver BC, Tony Bates Associates Ltd., ISBN: 978-0-9952692-0-0, 2015.

[9] H. Tinmaz, and J. H. Lee, "An analysis of users' preferences on learning management systems: a case on German versus Spanish students," Smart Learning Environments, vol. 7, 2020. https://doi.org/10.1186/s40 561-020-00141-8

[10] I. Witten, E. Frank, and M. Hall, "Data mining: Practical machine learning tools and techniques," Morgan Kaufmann Series in Data Management Systems Morgan Kaufmann, pp. 587-605, ISBN: 978-0-12 -374856-0 2011. https://doi.org/10.1016/B978-0-12-374856-0.00023-7.

[11] J. Cutler, and M. Dickenson, "Application programming interfaces," Computational Frameworks for Political and Social Research with Python, Springer, pp. 87-97, ISBN: 978-3030368258, 2020.

[12] J. R. Hipp, C. Bates, M. Lichman, and P. Smyth, "Using social media to measure temporal ambient population: Does it help explain local crime rates?," Justice Quarterly, vol. 36, issue 4, pp. 718-748, 2019.

[13] L. A. Bove, and S. Conklin, "Learning strategies for faculty during a learning management system migration," Online Journal of Distance Learning Administration, vol. 23, issue 1, pp. 1-10, 2020.

[14] L. Li, Zheng, F. Yang, and T. Li, "Modeling and broadening temporal user interest in personalized news recommendation," Expert Systems with Applications, vol. 41, issue 7, pp. 3168-3177, June 2014. https://doi.org/10.1016/j.eswa.2013.11.020.

[15] L. Yao, and Z. Ge, "Deep learning of semi-supervised process data with hierarchical extreme learning machine and soft sensor application," IEEE Transactions on Industrial Electronics, vol. 65, num. 2, pp. 14901498, 2018. https://doi.org/10.1109/TIE.2017.2733448

[16] M. A. Mohsen, and C. Shafeeq, "EFL teachers' perceptions on Blackboard applications," English Language Teaching, vol. 7, num. 1, pp. 108-118, 2014.

[17] P. Bradford, M. Porciello, N. Balkon, and D. Backus, "The Blackboard learning system," The Journal of Educational Technology Systems, vol. 35, issue 3, pp. 301-314, 2007.

[18] S. Gauch, M. Speretta, A. Chandramouli, and A. Micarelli, "User profiles for personalized information access," BRUSILOVSKY P., KOBSA A., NEJDL W. (dirs.), The Adaptive Web, Springer Berlin Heidelberg (Lecture Notes in Computer Science), pp. 54-89, 2007.

[19] S. On-At, M. F. Canut, A. Péninou, and F. Sèdes, "Time-aware egocentric network-based user profiling," Encyclopedia of Social Network Analysis and Mining, Springer $2^{\text {nd }} \mathrm{Ed}$, pp. 3113-3119, ISBN: 978-1-4939-7131-2, 2020.

[20] V. Aurélien, Intelligence artificielle vulgarisée: Le machine learning et le deep learning par la pratique, Éditions ENI, ISBN: 978-2-409-020735, 2020.

[21] Y. C. A. Padmanabha Reddy, P. Viswanath, and B. Eswara Reddy, "Semi-supervised learning: a brief review," International Journal of Engineering \& Technology, pp. 81-85, 2018. 